\title{
Über das Zungenbein der Reptilien
}

VON

\author{
MAX FÜRBRINGER.
}

Es ist mir eine grosse Ehre und Freude, der Festschrift, welche den \%0 jährigen Geburtstag meines lieben und hochgeschätzten Freundes Dr. Coenraad Kerbert ebren soll, auch meinerseits einen Beitrag beizufügen. Ich gedenke dabei der glücklichen und unvergesslichen Jahre, in denen ich mit der Universität Amsterdam verbunden war, in dem Garten und den Museen von N. A. M. so oft und gern verweilte und daselbst meine zoologischen Kenntnisse bereicherte, wo meinen zootomischen Untersuchungen zugleich durch die Güte des damaligen Directors, des hochverdienten Dr. G. F. Westerman, und meiner mit der Genootschap verbundenen Collegen und Freunde Dr. Coenraad Kerbert, Prof. Dr. Max Weber und K. N. Swikrstra eine so reiche Förderung mit Material zu Teil ward.

Aus den Arbeiten meiner letzten Jahre wähle ich diejenigen über das Zungenbein der Reptilien aus, für welche mir auch von. M. WEBER und C. KERBERT in jüngster Zeit zur Verfügung gestellte Tiere Gewinn und Untersuchungsmaterial brachten, und ich befinde mich hierbei als bescheidener Mitarbeiter zugleich auf dem gleichen Forschungsgebiete mit den drei hervorragenden holländischen Zoologen Prof. Eug. Dubois, Prof. Jan Verslurs und Prof. P. N. van Kampen, welche durch ihre in dem anatomischen (Dubois) und dem zoologischen (Versluys und van Kampen) Institute der Universität Amsterdam ausgeführten Untersuchungen für die wissenschaftliche Erkenntnis dieses Organes so Bedeutungsvolles, zum Teil selbst Bahnbrechendes geleistet haben.

Die Ungunst der Zeitverhältnisse hat es mir leider nicht erlaubt, meine bezüglichen Untersuchungen in ihrem ganzen Umfange und in gleichmässiger Bearbeitung zum Abschlusse zu bringen. So kann ich für jetzt zu meinem Bedauern nur einen kurzen, an bereits Bekanntes anknüpfẹden und nicht viel Neues darbietènden Auszug des ersten Abschnittes mitteilen, indem ich zugleich der Hoffnung Ausdruck gebe; dass es mir noch vergönnt sein möge, die ausführlichere, mit Abbildungen versehene und der älteren und neueren Literatur besser Rechnung tragende Arbeit in absehbarer Zeit zu veröffentlichen. Von einer Berücksichtigung der ausgestorbenen Reptilien muss hier in der Hauptsache abgesehen werden.

\section{DAS HYOID DER LEBENDEN REP'TILIEN.}

Das Zungenbein der lebenden Reptilien besteht in seiner vollkommensten Ausbildung, wie seit langer Zeit bekannt ist, aus einem unpaaren Körper, Corpus (Copula, Basihyale und Basibranchiale), der sich nach vorn in einen schmäleren in den hinteren Teil der Zunge einragenden Fortsatz, Processus lingualis (Pr. entoglossus), verjüngt, und drei, seitlich mit dem Körper in verschiedener Weise verbundenen Hörnerpaaren, einem vorderen, dem Corna hyale, einem mitt]eren, dem Cornu branchiale I, und einem hinteren, dem Cornu branchiale II. 
Das Cornu hyale, dem ursprünglichen Zungenbeinbogen entstammend, zeigt bei den primitiveren Formen Verband mit dem auch zu dem Hyoidbogen gehörigen columellaren Apparat des Mittelohres, hat aber diesen bei der überwiegenden Mehrzahl der Reptilien aufgegeben und ist bei vielen in eine weitgehende Rückbildung getreten. Das dorsal frei am Halse endende Cornu branchiale $l$. gehört dem 1. Branchialbogen (3. Visceralbogen) an und weist sich durch seine in der Regel vorhandene Verknöcherung, auch da, wo alle anderen Teile des Zungenbeins ihre Knorpelstructur beibehalten haben, sowie seine Beständigkeit als leistungsfähigster Teil des Hyoides aus. Das aus dem 2. Branchialbogen (4. Visceralbogen) hervorgegangene Cornu branchiale 1I. zeigt bei der Mehrzahl der Reptilien eine weit vorgeschrittenere Reduction mit häufigem Zerfall in einen ventralen und dorsalen Teil und kaun bei nicht wenigen Reptilien in völligen Schwund treten. Auch dieses Horn endet in der. Regel, dorsal frei, ist aber in gewissen vereinzelten Fällen mit dem dorsalen Ende des Cornu hyale verbunden.

Hinsichtlich eines.genaueren Übeŗblickes sei namentlich auf GAUPP's ausgezeichnete zusammenfassende Bearbeitungen der Ontogenese und Phylogenese des schallleitenden Apparates bei den Wirbeltieren (1899), der Entwicklung des Kopfskeletes (1905) und des Hyobranchialskeletes der Wirbeltiere (1905) verwiesen.

Das Zungenbein bildet zugleich in wechselnder Weise die ventrale Unterlage für die Luftwege (Larynx bezw. Cricoid und Trachea).

\section{Rhynchocephalia.}

Das Hyoid des einzigen noch lebenden Vertreters dieser Ordnung, Sphenodon punctatus, ist uns namentlich durch die Untersuchungen von Cope (92), Gegenbaur (98), Osawa (98) und Schauinsland (00, 03) bekannt geworden. Der Verband mit dern columellaren Apparat hat insbesondere Günther: (67), Huxley (69), Peters (70), Baur (87), Gadow (88), Killian (90) und vor Allen Versluys (98, 03), Schauinsland (00, 03) und Howes and Swinnerton (01) beschäftigt, wie auch Schauinsland, sowie Howes and Swinnerton hinsichtlich der Ontogenese hervorgehoben seien. Göppert $(99,01)$ untersuchte den Kehlkopf genauer.

Es besteht mit Ausnahme des grösstenteils verknöcherten Cornu branchiale I. durchweg aus Knorpel, der bei ausgewachsenen Tieren zu einem nicht geringen Teile verkalkt ist und ein auffallend dickes Perichondrium besitzt. Der Körper mit dem ganz allmählich aus ihm hervorgehenden Proc. linqualis tritt gegenüber den Hörnern durch Massivität hervor und liegt dem Larynx und den ersten Trachealringen auf, wobei der eigentliche Körper (excl. Proc. lingualis) den 3. und 4. Trachealring von unten deckt. Das Cornu hyale ist das bedeutendste und längste Horn und steht, von individuellen Ausnahmen abgesehen, mit der Extracolumella in continuirlichem Verband, welcher durch die embryologischen Untersuchungen von Schauinsland und Howes und Swinnerton als ein ursprünglicher erwiesen wurde. Auch lässt sich an ihm ein kleinerer mit dem Corpus verbundener ventraler und ein grösserer in die Extracolumella übergehender dorsaler Schenkel unterscheiden, welche sich im rechten Winkel treffen, ursprünglich separat angelegt sind, im weiteren Verlaufe der Entwickelung aber mit einander verschmelzen. Alles primitive Züge. Das knöcherne Cornu branchiale l. ist ansehnlich und mit kurzer Knorpelepiphyse versehen. Vom knorpeligen Cornu branchiale II. war bisher nur der lange, mit dem Hinterrande des Körpers continuierlich verbundene ventrale Teil bekannt; doch besitzt Sphenodon auch einen dorsalen T'eil, der ganz entfernt von dem ventralen in der Nähe des dorsalen Endes des Cornu branchiale I. sich befindet und einen sebr feinen, fast mikroskopischen und in dichtes Bindegewebe eingehüllten Knorpelstab, wahrscheinlich als Rest einer früheren voluminöseren Entwickelung, bildet.

Das Hyoid von Sphenodon zeigt einen durchaus lacertilen Bau und ist dem gewisser Agamidae (insbesondere Phrynocephalus) sehr ähnlich, wenn auch plumper als bei diesen gestaltet.

Bekanntlich betrachtet die Mehrzahl der Zoologen die Rhynchocephalia nach dem Vorgange von Günther $(6 \%)$ als besondere Ordnung in der Nähe des Ordo Lacertilia. Einige, z. B. Cope ( $70,87,89$, nicht aber 64 und 92), Seeley (74) und Boulenger (89) näherten sie den Cheloniern. Andere dagegen, unter ihnen Cope $(64,92)$, Peters $(\%)$, Huxley $(\% 1,8 \%, 91)^{\prime}$ Osawa $(98,99)$, Beddard (05), reihten sie den Lacertiliern ein oder fanden bei ihnen viele 
an diese Ordnung erinnernden Merkmale. Ich bin Anhänger der besonderen Stellung von Sphenodon als letzter lebend zurückgebliebener. Vertreter des O. Rhynchocephalia, muss aber hinsichtlich des Hyoides (und anderer.Verhältnisse ihres Baues) ihre sehr nahe Stellung zu den Lacertiliern betonen. Mit dem Zungenbein der Chelonier zeigt das Hyoid von Sphenoden gar keine Ähnlichkeit.

\section{Lacertilia (Sauria).}

Der Ordo Lacertilia wurde bekanntlich von Stannius (56) in die drei Unterordnungen der Amphisbaenoidea, Kionokrania und Chamaeleonidea eingeteilt, später von Boulenger $(84,85-87)$ in die beiden Subordines Lacertilia vera und Rhiptoglossia (= Chamaeleonidea), indem er die Amphisbaenoidea als einfache Familie Amphisbaenidae neben der Fam. Teiidae den Kionokrania einreihte und den ganzen Familiencomplex derselben incl. die Amphisbaenidae als S0. Lacertilia vera aufstellte. Versluys $(98,03)$ wurde andererseits durch seine Untersuchungen über das Verhalten des hyalen. Hornes zum columellaren Apparat dazu geführt, den Amphisbaenen wieder einen mehr separaten Platz im System anzuweisen, und auch andere Merkmale deuten auf eine besondere Stellung derselben hin, wenn auch die von Boulenger behauptete Verwandtschaft mit den Teiidae ihre guten Grundlagen hat. Unter den Lacertilia vera sind nach dem Boulenger'schen, inzwischen um eine weitere Familie vermehrten System 21 Familien zu unterscheiden, von welchen die Geckonidae, Eublepharidae und Uroplatidae die primitivsten repräsentieren; die hochstehenden und einseitig spezialisierten Rhiptoglossa enthalten nur die eine Fam. Chamaeleontidae.

Das Zungenbein der Lacertilier ist von zahlreichen Forschern untersucht worden, von denen ist hier nur Cuvier (24, 36, 38), Meckel (29), E. Geoffroy St. Hilaire (32), Stannius (56), Calori (57, 58, 61), Salverda (63), Leydig (72), Peters : (74, 82), Ficalbi (82), Smalian (87), Walter (87), Hoffmann (89, 90), Cope (92), Versluys (98, 03), Gaupp $(99,05)$, Beddard $(05,07)$ und Zavattari $(08,10)$ nenne; durch Anzahl der untersuchten Arten treten Zavattari, namentlich aber Cope unter ihnen hervor. Die Entwickelungsgeschichte wurde von W. K. Parker (80, 81), Gaupp (98) und vornehmlich Kallius (01) und Versluiis (03) behandelt. Hinsichtlich des Verhaltens des dorsalen Endes des Corru hyale zum Mittelohr sei auf die hervorragenden Untersuchungen von Versluys (98, 03), sowie die von Cords (09) und Zimmermann (11) hingewiesen. Über die Zunge und ihre Muskulatur handeln vornehmlich Brücke (52), Minot (80), Ludwig Ferdinand Prinz von Bayern (84), Kathariner (94), Oppel (00), Kallius (01), Göppert (03), Gandolf (08), über den Kehlkopf (Cricoid und Nebenknorpel) Meckel (33), Henle (39), Wiedersheim $(76,86)$, Eug. Dubois (86), Göppert (99, 01), Tornier (04-05), Werner (11), Germershausen (13), V. Schmidt (13) und H. Böker (18). In der Naturgeschichte der Zunge und des Kehlkopfes spielt Chamaeleo die Hauptrolle.

Ich wal in der glücklichen Lage, über eine nicht geringe Zahl von Arten, von denen ich einen grossen Teil der Güte von Prof. MAX Weber aus den Sammlungen von N. A. M. verdanke, zur Untersuchung zu verfügen. Auf Grund der bisherigen und meiner Beobachtungen gewann ich danach Kenntnis über alle Familien der Lacertidae, mit einziger Ausnahme der Ophiosepsidae, und zwar von 150 Arten (von denen nicht wenige in mehreren Exemplaren), die sich in die einzelnen Familien folgendermassen verteilen:

$$
\text { I. Lacertilia vera. }
$$

1. Geckonidae (24 Arten).

2. Eublepharidae (4)

3. Uroplatidae (1)

4. Pygopodidae (1).

5. Agamidae (18)

6. Iguanidae $(20)$

7. Xenosauridae (1)

\author{
8. Zonuridae (5) \\ 9. Anguidae (7) \\ 10. Anniellidae (1). \\ 11. Helodermatidae (2) \\ 12. Varanidae (4) \\ 13. Xantusiidae (2) \\ 14. Teiidae (7).
}

II. Rhiptoglossa.

22. Chamaeleontidae (11)
15. Amphisbaenidae (8).

16. Lacertidae (11)

17. Gerrhosauridae (2)

18. Scincidae (19)

19. Anelytropidae (1).

20 Ophiosepsidae $(0)$

21. Dibamidae (1).

Wie bereits oben bei den Rhynchocephalia angegeben, ist das Hyoid der Lacertilier nach dem gleichen Typus wie bei Sphenodon, aber schlanker gebaut. Dies gilt für den S0. Lacertilia vera (Fam. 1-21). Der SO. Rhiptoglossa (Fam. 22) weicht mehr ab und soll erst nach Besprechung der Lacertilia vera behandelt werden.

\section{a. Lacertilia vera (Kionokrania und.Amphisbaenia).}

Das im Grossen und Ganzen recht gracile Hyoid der Lacertilia vera besteht mit Ausnahme des Cornu branchiale I. durchweg aus Knorpel, der bei gewissen Vertretern verkalkte Stellen aufweist. Alle Knorpelteile stehen in der Regel in continuirlichem Zusammenhange; doch kommt es vereinzelt auch zu Abgliederungen und Verbindungen durch Faserknorpel oder faseriges Bindegewebe, selbst zu völligen Trennungen der Teile.

Das Corpus repräsentiert wie bei Sphenodon mit dem vorn von ihm abgehenden Proc. lingualis den mittleren unpaaren Teil. Es bildet eine der Trachea ventral aufliegende quere oder im Winkel nach vorn (rostralwärts) : strebende Brücke, welche in dieser Hinsicht, sowie in Länge und Breite recht wechselnde Verhältnisse aufweist und gegenüber den von ihm 
abgehenden paarigen Hörnern weniger in den Vordergrund tritt als bei Sphenodon; doch zeigen hier einige Vertreter, inshesondere bei den Agamiden, an Sphenodon erinnernde Verhältnisse. In jungen embryonalen Stadien geht der Körper nach Kallius' Untersuchungen an Lacerta muralis (01) in einen schmäleren hinteren Fortsatz (Urostyl) aus, der aber noch vor der Verknorpelung des Zungenbeins wieder verschwindet. Der schlanke Proc. 'lingualis kann, namentlich bei nach vorn strebendem Corpus, ähnlich wie bei Sphenodon ganz allmählich aus dem Körper hervorgehen; bei der Mehrzahl der Lacertilia vera setzt er sich unter plötzlicher Verschmälerung des Körpers scharf von diesem ab und bildet einen verschieden langen, schlanken, in den hinteren Teil der Zunge eindringenden Knorpelstab. Aus den betreffenden Dimensionen lassen sich keine systematischen Schlüsse ziehen; doch sei erwähnt, dass die Mehrzahl der Geckonidae, Eublepharidae, Uroplates, Lialis, die Zonuridae, die meisten Anguidae, Heloderma, die Lacertidae und meisten Scincidae durch recht lange, dagegen einzelne Agamidae und Iguanidae und die Teiidae durch recht kurze.Proc. linguales aus dem Heer der Formen mit mittellangen Ziungenfortsätzen sich hervorheben.

Das Cornu hyale (Cornu principale Gaupp) bildet in morphologischer und systematischer Hinsicht den wichtigsten Teil des Hyoides. Zusammen mit dem columellaren Apparat des Mittelohres aus dem ursprünglichen Hyoidbogen hervorgegangen, welcher Zusammenhang durch die Entwickelungsgeschichte bei verschiedenen Lacertiliern (einigen Geckonidae, Calotes und Lacerta) namentlich von VersLUYs als ein, ursprünglicher nachgewiesen und bei Amphisbaena vom gleichen Autor sehr wahrscheinlich gemacht wurde, hat es diesen primordialen Verband entweder unter Bildungshemmung bei Amphisbaena bewahrt oder unter Ablösung von der Extracolumella mit einem neuen benachbarten und in der Hauptsache wohl auch vom columellaren Apparate abstammenden Verbande mit dem Proc. paroticus, sowie der daneben befindlichen der Labyrinthwand angehörigen Crista parotica vertauscht (Geckonidae, Eublepharidae, Uroplates) oder bei der ganz überwiegenden Mehrzahl der Lacertilier ganz aufgegeben und damit eine Lage seines frei gewordenen dorsalen Endes am Anfange des Halses gewonnen. Danach nimmt kein Lacertilier eine so primitive Stellung ein wie gewisse Exemplare von Sphenodon; jedoch die drei Familien der Geckonidae, Eublepharidae und Uroplatidae, welche auch durch andere Merkmale ihres Baues sich als die am tiefsten stehenden Lacertilier zu erkennen geben, stehen ihm in dieser Hinsicht nicht so fern, während die anderen Lacertilierfamilien, darunter auch die Agamidae mit ihrem übrigens dem von Sphenodon recht ähnlichen Zungenbein, in dem Verluste des Verbandes des Cornu hyale mit dem Columellarapparat sich von den Rhynchocephaliern weiter ab stellen. In wie fern es ursprüngliche Vorfahren von ihnen gegeben hat, die mit Sphenodon in diesem Stücke eine grössere morphologische Gemeinschaft zeigten, ist eine Frage, die wohl aufgeworfen, zur Zeit aber nicht mit Tatsachen beantwortet werden kann. Bei allen mit einem wohl entwickelten Hyalhorn versehenen Lacertiliern ist, wie bei Sphenodon, ein kürzerer ventraler und ein längerer dorsaler Schenkel (Teil) zu unterscheiden. Ersterer erstreckt sich von dem lateralen Ende des Vorderrandes des Körpers, in der Regel continuierlich mit ihm verbunden, lateral- oder lateralrostral- oder rostralwärts nach der Seite und (oder) nach vorn und geht nach kurzem Verlaufe am vorderen Scheitelpunkte unter einem meist spitzen oder rechten, seltener stumpfen bis gestreckten Winkel in den dorsalen Schenkel über, der in caudaler und (oder) lateraler und schliesslich dorsaler Richtung verläuft und, wie schon angegeben, entweder im Mittelohr am columellaren Apparat oder Schädel sich befestigt oder frei am Halse endet, wobei er in der Regel von dem Musc. episterno-cleido-mastoideus bedeckt wird, bei manchen Lacertiliern auch einem Teil dieses Muskels als Ursprung dient (VErsLurs 98, was ich bestatigen kann). Der ventrale Schenkel ist nicht allein der kürzere, sondern meist auch der schmälere, schwächere und einfacher gebaute Teil des Cornu hyale, der dorsale wiegt bei guter Ausbildung des hyalen Horns nicht nur an Länge, sondern auch in den anderen Dimensionen und in den sonstigen Complicationen seines Verlaufes und seiner Configuration mehr oder minder bedeutend über den ventralen' vor. Hier finden sich viele für die kleineren Abteilungen taxonomisch verwertbare Züge. Gewisse Familien, insbesondere die Zonuridae, Lacertidae und namentlich 
Scincidae kennzeichnet eine zum Teil recht beträchtliche lateralwärts gehende Verbreiterung mit lateraler Verdünnung des vorderen Abschnittes; andererseits zeigen die Anguidae und Heloderma eine mässige Verbreiterung des hinteren Bereiches. Weitere Differenzierungen anderer Art, die sich indessen nicht in Kürze wiedergeben lassen, sind bei verschiedenen Familien. in mannigfacher Weise zu beobachten. Der Scheitelpunkt des Cornu hyale ragt bei den ausgewachsenen Tieren unter Ausbildung des rechten oder spitzen Winkels in der Regel mehr. oder minder weit rostralwärts über die beiden Schenkel vor. Die von Kallius (0I) des Genaueren geschilderte Ontogenese zeigt, dass das hyale Horn in frühen Stadien von Anfang an einen lateralen Verlauf ohne winkelig hervortretenden Scheitelpunkt darbietet und dass erst bei der weiteren Entwickelung die im Winkel sich treffenden Schenkel zur Unterscheidung kommen. Sphenodon mit seinem lateralwärts gerichteten ventralen Schenkel off $n$ bart auch darin ein relativ primitives Gepräge. Die Ausbildung geht Hand in Hand mit der Verlängerung der beiden Schenkel und der Behinderung einer sonstigen seitlichen Ausdehnung derselben, ein Vorgang, der auch bei den Anuren Analogien zeigt, in dem Causalnexus seiner eigentlichen Genese und Correlation aber noch nicht genügend aufgeklärt ist. Bei einzelnen Lacertiliern finden sich Combinationen rein lateralen bis lateral-caudal gebenden Verlaufes mit unter gestrecktem Winkel, d.i. in gerader Linie sich treffenden beiden Schenkeln (Coleonyx, Draco, Phrynosoma), die etwas an embryonale Verhältnisse erinnern, aber nur als secundäre Differenzierungen aus dem bei den Meisten vorkommenden Verhalten zu erklären sind. Auch der Scheitelpunkt, der bei der Mehrzahl der Familien als einfacher Übergang des ventralen in den dorsalen Schenkel auftritt, kann besondere Gestaltungen in Form mannigfacher Vorragungen darbieten, so namentlich bei den Geckonidae und Eublepharidae, und in anderer Weise bei Uromastix ornatus, Xenosaurus, den Anguidae, Varanus und insbesondere den Teiidae. Ihre besondere systematische Stellung bekunden die Varanidae auch darin, dass bei ihnen am Scheitelpunkte die beiden Schenkel völlig von einander getrennt bezw. in eine ventrale und dorsale Lage gespalten sind, wobei der dorsale Schenkel an seinem Beginne dem rostralen Ende des ventralen Schenkels ventral aufliegt. - Bei nicht wenigen Vertretern tritt das Cornu hyale in eine Rückbildung, die im Bereiche des dorsalen Schenkels beginnt, weiterhin zu dessen völliger Reduction führt und schliesslich den ventralen Schenkel ergreift, verkürzt und endlich auch zum vollkommenen Schwunde desselben und damit des ganzen hyalen Horns führt. Eine derartige Reduction des dorsalen Schenkels unter Persistenz des ventralen und danach des ventralen Schenkels kommt in fortschreitendem Grade bei gewissen Arrguidae, bei Lialis, den Amphisbaenidae, Acontias unter den Scincidae, sowie Typhlosaurus, vielleicht auch individuell bei Anguis zur Beobachtung, bei Acontias und Typhlosaurus unter beginnender und weiter fortschreitender Verkümmerung des ventralen Schenkels. Bei Anniella (Cope 92, Baur 94, Kunkel 05) und bei Dibamus (eigene Untersuchung) ist der letzte Rest des Cornu hyale geschwunden. Die Rückbildung findet somit in verschiedenen Familien statt, die sich zum Teil verwandtschaftlich nicht nahe stehen, ergreift aber mit Vorliebe diejenigen Vertreter derselben, die unter Verlust ihrer Gliedmassen und unter secundärer Verlängerung. ihres Körpers eine schlangenartige Gestalt angenommen haben.

Das in seiner hauptsächlichen Ausdehnung verknöcherte und an seinem freien Ende in eine knorpelige Epiphyse auslaufende Cornu branchiale I. bildet den leistungsfähigsten und in seiner Existenz beständigsten Teil des Zungenbeines. Es ist in der Regel gelenkig mit dem Seitenrande des knorpeligen Körpers verbunden, von mässig wechselnder, gewöhnlich das Hyalhorn übertreffender länge und von variierendem Verlaufe (geradlinig; gestreckt bogenförmig, gestreckt Sförmig) in einer im Allgemeinen caudal-lateral-dorsalwärts gehenden Richtung, wobei bald diese bald jene Direction überwiegt. Die Epiphyse wechselt in Länge, Richtung und Gestalt, ohne besondere taxonomisch verwertbare Directiven zu gewähren. Wie oft sie auch nicht abgebildet worden, so fehlt sie doch wohl niemals. Mit dem Ende des Knochenteils liegt sie dem Musc. episterno-cleido-mastoideus oberflächlich auf, zeigt sonach in ihrer Beziehung zu diesen Muskel ein recht abweichendes Verhalten vom Cornu hyale. Bei Draco springt das Cornu branchiale I, oberflächlich hervor und bildet gemeinsam mit 
dem direct neben ihm verlaufenden Cornu hyale die Grundlage der paarigen seitlichen Kehlfalte dieses Tieres.

Das knorpelige Cornu branchiale II. tritt in seiner functionellen Leistungsfähigkeit mehr oder minder erheblich hinter dem Cornu branchiale I. und meist auch dem Cornu hyale zurück, ist auch derjenige Teil des Zungenbeins, welcher im Allgemeinen dem Reductionsprocess am frühesten und ausgiebigsten unterworfen ist. Dafür besitzt es eine morphologische und zum Teil taxonomische Bedeutung, welche die des.1. Branchialhorns bei weitem übertrifft und der des Hyalhorns nur wenig nachsteht. Die auf dasselbe untersuchten Lacertilier bilden zugleich eine vergleichend anatomische Entwickelungsreihe, welche in glücklicher Weise den Rückbildungsgang dieses Hornes aufhellt und manche früher des Zusammenhanges beraubten und daher unverständlich gebliebenen Verhältnisse klarlegt. Den Anfang dieser Reihe bildet Coleonyx elegans, Vertreter der Eublepharidae, der (nach Cope's Abbildung 92) ein langes von dem lateralen Bereichè des Hinterrandes des Körpers obne Grenze abgehendes 2. Branchialhorn besitzt, das zuerst caudalwärts nach hinten, dann im Bogen lateralwärts und dorsalwärts und endlich rostralwärts sich nach vorn wendet, wobei es das dorsale Ende der 1. Branchialhornes, von diesem bedeckt, kreuzt und der Innenfläche des ventralen Randes des Musc. episterno-cleido-mastoideus anliegt; schliesslich endet es, im dorsalen Bereiche des Halses nach vorn verlaufend, in der Nähe der dorsalen Schenkel des Hyalhornes. Dieses Gebilde fasse ich als das gesamte einheitliche Cornu branchiale II. auf, wobei noch daran zu denken ist, ob nicht der letzte, in longitudinaler Richtung nach vorn verlaufende Teil einer Commissura terminalis (Gaupp 93), wie sie in allerdings abweichender Weise bei urodelen Amphibien beobachtet wird, zu vergleichen sei. Bei den anderen von mir auf das 2. Branchialhorn untersuchten Eublepharidae, Psilodactylus caudicinctus und Eublepharis macularius, hat eine Sonderung dieses gemeinsamen Hornes in einen ventralen und dorsalen 'I'eil stattgefunden, beide lange, sich noch berührende Abschnitte, welche an der Berührungszone eine kleine Strecke lang neben einander liegen; und die gleiche Sonderung und Bildung bieten Cope's Abbildungen (92) der beiden Vertreter der Fam. Xantusiidae, Xantusia riversiana und Lepidophyma flavomaculatum dar, wobei zugleich bei letzterer Art das dorso-rostrale Ende des dorsalen Teiles noch eine secundäre Verbindung mit dern Basioccipitale eingegangen ist. Bei allen anderen Lacertilia vera sind beide Teile, soweit sie bei ibnen überhaupt noch vorhanden, namentlich der dorsale, mehr verkümmert und mit ihren ursprünglich benachbarten Enden auseinander gerückt; doch weist insbesondere bei den Scincidae der caudallateralwärts gehende Verlauf des ventralen Teiles noch auf die Richtung hin, wo der dorsale Teil sich befindet. Es ist bemerkenswert, dass die wenig verwandten Eublepharidae und Xantusiidae im Wesentlichen die gleichen Bildungen darbieten und dabei zugleich von den (den Eublepharidae nahe verwandten und tiefer stehenden) Geckonidae, bei denen der ventrale und dorsale Teil weit mehr verkümmert sind, quantitativ so erheblich abweichen. Weitere Untersuchungen, namentlich zur Entscheidung der Frage, ob hier auch an secundäre Convergenzen zu denken sei, sind wünschenswert. Der ventrale Teil (Cornu branchiale II. ventrale) zeigt von einer noch ansehnlichen Ausbildung bis zu einer weiter fortgeschrittenen Rückbildung und bis zum völligen Schwunde alle möglichen Zwischenstufen, selbst innerhalb der Familien, z. B. der Geckonidae, Zonuridae und Scincidae, bei denen gut entwickelte, mässig grosse und kleine, zum Teil nur mikroskopisch nachweisbare ventrale Teile sich finden, die continuirlich von dem hinteren Rande des Corpus ausgehen und vorwiegend an den beiden Seitenrändern der Trachea in caudaler Richting verlaufen. Bei verschiedenen Geckonidae, bei Uroplates, Xenosaurus, den Anguidae, Anniella, Heloderma, Varanus, den meisten Teiidae, Rhineura (Cope), Gerrhosaurus (Cope), einzelnen Scincidae, darunter namentlich Acontias, ferner Typhlosaurus und Dibamus, fehlen sie ganz, sind dagegen vornehmlich bei vielen Vertretern der Lacertidae und Scincidae noch sehr gut ausgebildet. Hierbei sind die mit wohlentwickelten Extremitaten versehenen eidechsenartigen und die der äusseren Gliedmassen entbehrenden schlangenähnlichen Lacertilier zu unterscheiden. Bei den Ersteren kennzeichnet die tiefer stehenden Familien eine noch gute Persistenz des Cornu 
branchiale II. ventrale, die höheren eine vorgeschrittene Rückbildung desselben bis zum völligen Schwund; bei den Letzteren verbindet sich mit der schlangenartigen Ausbildung des Körpers in der Regel ein mehr oder minder vollkommener Ausfall dieses ventralen Teiles. Ein besonderes Verhalten bieten die beiden nahe verwandten Familien der Agamidae und Iguanidae dar. Hier findet sich, wie schon seit lange bekannt, aber im Detail nicht näher verfolgt worden, eine bedeutende Variirung der Länge. und Lage des rechten und linken Cornu branchiale II. ventrale: beide verlaufen bei mässiger Ausbildung von einander entfernt zu beiden Seiten der Luftröhre, kommen bei Vergrösserung ihrer Länge einander näher und treten schliesslich bei noch grösserer Länge, welche sie über den grösseren Teil des Halses und selbst über den Anfang der Brust hinabreichen lässt, zu einander in direkte Berührung. Hierbei stützen sie zugleich die mittlere Kehlfalte. Es ist klar, dass es sich bei dieser eigenartigen Verlängerung um kein Zurückkehren zu primitiveren Zuständen, sondern um eine einseitige secundäre Differenzierung handelt. - Der dorsale Teil des 2. branchialen Hornes (Cornu branchiale II. dorsale) bildet - mit Ausnahme der oben erwähnten Eublepharidae und Xantusiidae - bei der Mehrzahl der Lacertilia vera nur ein untergeordnetes, in der Rückbildung ziemlich vorgeschrittenes Gebilde. Doch kennt man seit Cuvier (24), Parker (" 9 ), Cope (92), Gaupp (98), Beddard (07) und Zavattari (08) einige wenige Zonuridae (Zonurus), Anguidae (Gerrhonotus), Lacertidae (Lacerta) und Scincidae (Trachysaurus, Tiliqua, Lygosoma, Eumeces, Scincus), bei denen deutlich, bei Lacerta individuell sogar recht ansehnlich entwickelte Reste dieses dorsalen Teiles beobachtet wurden. Ich konnte diese Befunde grösstenteils bestätigen und fand auf Grund von gar nicht sehr eingehend und an einem nicht immer tadellosen Material durchgeführten Untersuchungen eine viel weitere Verbreitung dieses Cornu branchiale II. dorsale bei den Geckonidae, Uroplates, Uromastix und einigen anderen Agamidae und Iguanidae, Zonurus, den Anguidae; Heloderma, Tupinambis, Lacertidae, Zonosaurus und Scincidae in den verschiedensten Abstufungen der Grösse, Gestalt und Lage bis herab zum mikroskopischen Befunde, und ich bezweifle nicht, dass geflissentlich auf seine Existenz angestellte Beobachtungen es noch bei vielen anderen Lacertiliern werden finden lassen. Auch zeigt sich eine deutlich ausgesprochene Selbständigkeit in der Ausbildung und Rückbildung des ventralen und dorsalen Teiles: oft entsprechen sie sich hierin gegenseitig, es kann aber auch vorkommen, dass der ventrale noch bésser entwickelt ist als der dorsale oder dass das gegenteilige Verhalten sich findet.

Die Lage des Hyoidkörpers sensu strictiori (excl. Processus lingualis) zeigt den Luftwegen gegenüber einen grossen Wechsel. Bei Dibamus entspricht sie der des Kehlkopfes (Cricoid), bei den meisten Geckonidae, Eublepharidae und Uroplates, sowie bei Acontias vornehmlich der der ersten Trachealringe und zum Teil des hinteren Endes des Cricoid, bei den meisten anderen Lacertilia vera liegt er in mannigfachen Varianten dem 4. bis 9., bei Varanus dem 22. bis 25. Trachealringe an. Kehlkopf und Trachea finden sich somit bei den am tiefsten stehenden Familien, sowie bei gewissen schlangenähnlichen Lacertiliern im Verhältnis zum Hyoid weiter hinten als bei den höheren Familien derselben. Dies bildet auch eine gewisse vergleichend anatomische Parallele zu den ontogenetischen Befunden von Kallius (01), der bei seinen jüngsten embryologischen Stadien von Lacerta muralis den Aditus laryngis ziemlich weit hinter (caudal von) dem Hyoidkörper, noch caudal von der vergänglichen hinteren Fortsetzung (Urostyl) desselben, im nächsten Stadium am hinteren Rande des Körpers, in einem noch älteren Stadium in seiner Mitte und beim ausgebildeten Tiere rostral vor dem Körper beobachtete. In der von mir angegebenen Reihe dürften die am tiefsten stehenden eidechsenartigen Saurier die ursprünglicheren Verhältnisse wiedergeben; wăhrend die schlangenartigen Gattungen Acontias und Dibamus vermutlich auf dem Wege einer secundären Rückwanderung der Luftwege zu ihrer jetzigen Lage des Cricoides gekommen sind. Varanus mit seinem sehr weit rostralwärts vorgerückten Kehlkopfe steht ganz ausserhalb der übrigen Reihe und bezeugt auch in diesem Punkte seine besondere Stellung innerhalb der Lacertilia, auf die verschiedene Autoren und ich selbst in früheren Veröffentlichungen hinweisen konnten und die ihn selbst zu einer besonderen, dèn Mosasauria nahe stehenden Unterordnung erheben liess. 


\section{b. Rhiptoglossa (Vermilinguia, Chamaeleontia).}

Die dem Subordo Rhiptoglossa entsprechende Familie Chamaeleontidae mit ihren nahe benachbarten drei Genera Chamaeleo, Brookesia und Rhampholeon hebt sich in der Bildung ihres Zungenbeins, die bei allen drei Gattungen im Wesentlichen die gleiche ist, mehr von den Lacertilia vera $a b$, als diese von Sphenodon.

Dies liegt vornehmlich an der besonderen Configuration des Hyoidkörpers und des Proc. lingualis, die zufolge der eigenartigen Funktionierung der Zunge der Chamaeleonten ihre eigentümliche Bildung erhalten haben. Der Proc. lingualis hebt sich nicht vom eigentlichen Körper ab und bildet mit ihm einen langen und kräftigen cylindrischen Stab von oberflächlich stark verkalktem Knorpelgewebe, welcher der über ihn gestülpten und ihn umfassenden Zunge als centrale. Stütze dient und derselben zusammen mit der hochentwickelten Zungenmuskulatur und der synovialen ihn direkt umgebenden Scheide die bekannten, von zahlreichen Untersuchern eingehend beschriebenen Bewegungen ermöglicht. Wenn man, wie bei den Lacertilia vera, als eigentlichen Körper (Corpus sensu strictiori) denjenigen hintersten Teil des einheitlichen Stabes abgrenzt, von welchem die seitlichen Hörner abgehen, so ist der rostral vor ihın liegende Teil, der Proc. lingualis, 10 bis 15 mal länger und voluminöser als der eigentliche Körper, der eine besondere schwache ventrale Wölbung aufweist. Vorn und hinten endet das gemeinsame Gebilde stumpf.

Die beiden vom Körper, also am Ende des Stabes, abgehenden Hörnerpaare folgen dicht auf einander und treten in ihrer Grösse bedeutend gegen den Stab zurück. Das vordere Paar, Cornu hyale, besteht aus zum Teil verkalktem Knorpel und verlauft vom Körper aus überwiegend in lateral-dorsaler und ein wenig rostralwärts gehender Richtung, um ohne Verband mit dem Schädel oder dem columellaren Apparat frei zu enden. Hierbei weist es eine deutliche Gliederung auf, welche den kürzeren ventralen und den längeren dorsalen Teil (Schenkel), obwohl dieselben in gerader Linie (also in gestrecktem Winkel) verlaufen, doch gut von einander unterscheiden lässt; der dorsale Teil ist zugleich ein wenig schlanker als der mit dem Körper continuirlich verbundene ventrale und läuft dorsal spitz aus. - Das hintere Hörnerpaar, Cornu branchiale I., ist grossenteils verknöchert und hebt sich dadurch scharf und discontinuirlich vom Ende des Körpers ab. Ähnlich dem hyalen Horn verläuft es lateraldorsal mit einer geringen caudalwärts gehenden Neigung. Vermöge seiner Knochenstructur ist, es leistungsfähiger als das Cornu hyale, auch ein wenig länger und bei Chamaeleo ein wenig stärker oder ihm gleich stark, während ich es bei Brookesia und Rhampholeon eher etwas schwăcher als das hyale Horn finde. - Reste eines Cornu branchiale II. wurden weder im ventralen noch im dorsalen Bereiche gefunden. Eine sehr junge Stadien berücksichtigende ontogenetische Untersuchung steht noch aus; was Parker (80) darüber mitteilt, beschäftigt sich mit älteren Stadien, die vom ausgewachsenen Tiere keinen wesentlichen Unterschied aufweisen.

Der eigentliche Körper liegt in der Höhe des Kehlkopfes (Cricoid), diesen stützend; gleich caudal hinter ihm ragt der übrigens bei der Mehrzahl der Chamaeleontiden nicht zur Ausbildung gekommene Kehlsack hervor (vergl. hierüber namentlich Wiedersheim (86), Tornier (04, 05), Werner (11) und Germershausen (13)).

Das Zungenbein der Rhiptoglossa gibt sich in der Hauptsache nicht allein als eine eigenartige, sondern auch als eine höhere Bildung der Lacertilier zu erkennen, wie überhaupt der ganze Bau der Chamaeleontia sich überwiegend als Summe höherer und spezieller Differenzierungen darstellt. Das beherrschende Moment bildet hierbei der zur Zunge in direkterer Beziehung stehende Teil, und es ist bewundernwert, wie mächtig und tiefgreifend der Einfluss der Zunge und ihrer Bewegungen auf das ganze Zungenbein eingewirkt hat. Man könnte auch hier von Allmacht der Naturzüchtung sprechen. Dass die Anfänge der chamaeleontiden Bildung von einem nach Art der Lacertilia vera gebauten Hyoid abzuleiten sind, darf angenommen werden; ob eine rationelle, von den frühestẹ Stadien ausgehende embryonale Untersuchung hier Aufklärung geben wird, ist abzuwarten. Auf vergleichend-anatomischem 
Wege fand ich $(00)$ in anderen Organgebieten gewisse Ähnlichkeiten mit Uroplates und postulierte darauf eine natürlich nicht zu überschätzende Verwandtschaft mit diesem primitiven Saurier, derart, dass die Wurzel der Chamaeleonten sich in der Nähe derjenigen von Uroplates befunden haben möge. Boulenger (01) hat diesen Versuch zurückgewiesen, Werner (12) dagegen unter Herbeiführung weiterer Argumente gestützt. Das Hyoid ist der von Werner und mir angenommenen Verwandtschaft nicht günstig; zum Teil mögen auch secundäre Convergenzen von uns zu Gunsten einer Affinität verwendet worden sein. Weitere Untersuchungen erweisen sich als nötig.

\section{Ophidia.}

- Bekanntlich wird von den Zoologen und Paläontologen ziemlich allgemein und mit guten Gründen angenommen, dass Lacertilier und Ophidier relativ nahe Verwandte sind; beide werden auch von vielen Herpetologen als Ordnungen der Sectio. Streptostylica oder als Unterordnungen des Ordo Lepidosauria s. Squamata aufgefasst. Hierbei stellt man die Lacertilier (Saurier) als die Ausgang gebende Abteilung auf und lässt die Ophidier sich in einseitiger Weise von ihrer Wurzel abzweigen. Zugleich wurden früher nach JoH. MüLLER's Vorgange die in ihrer Kieferbildung den.Lacertiliern näher stehenden Angiostomata als primitivere, die von diesen ableitbaren Eurystomata als höhere Unterabteilung der Ophidier angenommen, wobei jedoch eine auf wirklich genetischer Grundlage ruhende Genealogie nicht nachweisbar war. Boulenger (93-96), dessen System mir übrigens nur durch Gadow's Bearbeitung der Amphibien und Reptilien (01) bekannt geworden, löst diese Unterabteilungen auf und statuiert 9 auf einander folgende Familien (s. unten), von denen die 4 ersten den Engmäulern und die 5 letzten den Weitmäulern Müllers entsprechen.

Das Zungenbein der Ophidier hat die Bearbeitung einer geringeren Zahl von Untersuchern gefunden als dasjenige der Lacertilier; ich nenne von diesen nur Cuvier (24, 38), Meckel (29), Joh. Müller (31), d'Alton (34), Losana (34), Rathke (39), Stannius und Peters (56), Leydig (83), Walter (87), Hoffmann (90), McKay (90), Gegenbaur (98), Göppert (99), hinsichtlich der Entwickelungsgeschichte Rathke (39), :Parker (78) und Peyer (12), und verweise im Übrigen auf die zusammenfassenden Darstellungen von Gaupp (05) und die bekannteren Lehrbücher der vergleichenden Anatomie. Auch die benachbarten Teile, Columellarapparat, Zunge und Kehlkopf, sind Objekte wiederholter Untersuchungen gewesen, von denen hier nur diejenigen von Henle (39), Minot (80), Ludwig Ferdinand Prinz von Bayern (84), Gadow (88), Göppert (99), Müller (05) und Okajima (15) angeführt seien.

Ich selbst habe nicht viele Schlangen bearbeitet und verfüge mit den in der Literatur angegebenen nur über Beobachtungèn an 31 Arten (verschiedene in mehreren Individuen), die sich nach den Familien folgendermassen verteilen:
1. Typhlopidae (2).
4. Uropeltidae (1).
5. Boidae (5).
7. Colubridae (12).
2. Glauconiidae $(0)$
3. Ilysiidae (2).
6. Xenopeltidae (1).
8. Amblycephalidae (0)
9. Viperidae (8).

Der Bau des Hyoides der Schlangen ist ein ungemein einfacher und von dem des Zungenbeines der Lacertilier erheblich abweichender, so dass aus ihm allein die Verwandtschaft beider Ordnungen nicht erschlossen werden könnte. In seiner bisher bekannten vollkommensten Ausbildung besteht es aus zwei mehr oder minder langen Knorpelfäden, einem rechten und linken, die längs der Trachea oder in ihrer Nachbarschaft verlaufen und vorn durch einen schmalen bogenförmigen und gleichfalls knorpeligen Querschenkel verbunden sind, welcher sich ventral über die Trachea legt, bei seiner Feinheit ihr aber nicht als Stütze oder Schutz dienen kann. Verknöcherte Teile fehlen ihm, soweit bisher bekannt, ganz, doch kann der Knorpel Verkalkungen darbieten. Mit dem Schädel hat das Zungenbein keine Verbindung, ist vielmehr bei seiner ventralen und weit nach hinten erstreckten Lage von ihm recht entfernt.

Es macht keine Schwierigkeit, die beiden Knorpelfäden als Hörnerpaar und den sie vorn verbindenden Querschenkel als Körper zu deuten; schwieriger ist die Frage zu beantworten, welchem Hörnerpaar der Lacertilier das der Ophidier entspricht.

Auf embryologischen Beobachtungen von Rathke (39), Parker (\%8) und Peyer (12) fussend, ist dasselbe als Cornu hyale aufgefasst worden, so auch von Gaupp (05), doch nicht mit Bestimmtheit, da die Angaben der Embryologen nicht lückenlos sind bezw. mit zu späten Stadien beginnen und über die bedeutende Umlagerung des von ihnen angegebenen Hyoidbogens nach hinten, die man annehmen muss, um zum wirklichen Vergleiche zu gelangen, keine genügende Auskunft geben. McKay (90) hat daher dieser Auffassung nicht zugestimmt und das Horn als Cornu branchiale II. gedeutet, wofür ihm der ventrale nach hinten gehende Längsverlauf neben der Trachea und der Verband mit der Muskulatur bestimmend war. Ich neige mehr einer Deutung als Cornu branchiale I. zu, worin ich auch einen Vorgänger in Losana 
(34) habe, der übrigens keine Beweise für seine Ansicht versucht. Diese Deutung lässt sich meiner Meinung nach mit dem Verlaufe des Hornes und mit seinen Muskelverbänden mindestens ebenso gut wie diejenige als Cornu branchiale II. vereinigen, und wird auch durch die Art seines Überganges in den vorn liegenden Hyoidkörper sowie durch die Erwägung gestützt, dass bei den Rückbildungen des Hyoides der Lacertilier, die sich bei deren schlangenähnlichen Typen finden, in extremen Fällen (Anniella, Dibamus) nur Körper (mit Proc. lingualis) und 1. Branchialhorn, also nach meiner Auffassung die gleichen Teile wie bei den Schlangen; persistieren; das 1. Branchialhorn bei den genannten. Lacertiliern allerdings als kürzeres und verknöchertes Gebilde. Dass bei durchgreifenden Reductionen die höhere Ossificationsstufe nicht erreicht wird und die Rückbildung, mit dem. Knorpelzustande Halt macht, ist indessen eine bekannte Erscheinung, und die bedeutende Verlängerung des Hyoidhornes der Ophidier steht mit der Verlängerung des gesamten Schlangenkörpers in Einklang, wobei auch natürlich nur als Beispiel für die hier bestehenden Wachstumsmöglichkeiten - an die mächtigen Verlängerungen des Cornu branchiale II. ventrale innerhalb der Agamidae und Iguanidae gedacht werden kann. Ich halte also zunächst das fragliche Horn der Ophidier für das Cornu branchiale I., erwarte aber erst von einer genaueren ontogenetischen Untersuchung die wirkliche Entscheidung.

Die relativ beste und am meisten normale Ausbildung des Zungenbeines findet sich, soweit bisher bekannt, bei den Colubridae und Viperidae. Hier verlaufen das rechte und linke Cornu branchiale $I$. in ansehnlicher oder bedeutender Länge annähernd parallel am lateralen Rande der Trachea, enden hinten frei und verbinden sich vorn $\mathrm{zu}$ dem ihnen in der Regel annähernd gleich starken, nicht selten auch etwas breiteren oder schmäleren Corpus, das mitunter in eine kürzere, schmal endende vordere Spitze als Andeutung des Processus lingualis ausläuft. Hierfür lässt sich eine förmliche Reihe aufstellen: Vipera ammodytes und danach Bungarus fasciatus mit leidlich langem Proc. lingualis, Naja haje und Tropidonotus natrix (individuell) ohne diesen, aber mit ziemlich breitem Körper, Tropidonotus natrix (individuell), Dipsas, Vipera aspis mit einem den Hörnern gleich starken Corpus und endlich Coronella austriaca (Leydig) mit schmälerem Körper. Die Länge der Hörner ist mässig bei Crotalus, ziemlich bedeutend bis sehr bedeutend bei der Mehrzahl der untersuchten Colubridae und Viperidae, vor Allen bei Bungarus (bei Vipera aspis-mass ich die Länge in der Ausdehnung von 35-36, bei Naja haje zu 5\%-59, bei Bungarus fasciatus zu 90-91 Trachealringen). Meist endeten die Hörner hinten nicht in gleicher Länge, auch zeigten sich zum Teil Abweichungen vom parallelen Verlaufe, indem sie bei Naja an ihrem caudalen Ende, bei Bungarus in einer längeren caudalen Strecke zur Berührung kamen und hier zugleich von der Mittellinie unter Drehung der Trachea seitlich abwichen. - Die untersuchten Xenopeltidae und Boidae zeigten einen von hinten nach vorn convergierenden Verlauf der mittellangen Hörner, die an ihrem caudalen Ende ziemlich oder recht weit von der Trachea abstanden und vorn entweder zu einem mässig breiten oder schmalen Körper sich vereinigten (Xenopeltis unicolor; Python reticulatus juv., ob individuell?) oder unter Schwund dieses Körpers auch vorn frei, aber benachbart endeten (Python molurus und P. sebae). - Eine noch grössere Rückbildung bieten die untersuchten Ilysiidae und Typhlopidae dar, indem bei ihnen die gleichfalls rostralwärts convergierenden und haarfeinen Hörner vorn auch nicht zur Berührung gelangten und namentlich bei den Typhlopidae von einer derartigen mikroskopischen Feinheit waren, dass über ihr vorderes Ende an den wenigen mir zur Verfügung stehenden und nicht gut erhaltenen. Tieren keine völlige Klarheit erlangt werden konnte; auch zeigten hier die Knorpelfäden eine Abwechselung von dünneren und dickeren Stellen, wohl Kennzeichen einer weit vorgeschrittenen 'Verkümmerung.

Die Lage der Luftwege zu dem Zungenbein ist eine aussergewöhnliche, indem erstere mit der bedeutenden Verlängerung der Trachea extrem weit nach vorn, mit dem Kehlkopf (Cricoid) und Aditus laryngis bis in den vorderen Teil des Mundes gerückt sind, das Zungenbein aber zurückgeblieben ist. So liegt der vordere Anfang des Hyoides immer um eine ansehnliche Anzahl von Trachealringen (bisher wurden von mir 9-36 gezählt) hinter dem 
Cricoid, ein Verhalten, welches die schon bei den Lacertiliern beobachtete Tendenz der laryngealen Vorwanderung noch übertrifft und meines: Wissens von keinem anderen Wirbeltier überholt wird; nur Varanus bietet in dieser Hinsicht eine ähnliche Lage innerhalb der von den Schlangen eingenommenen Grenzen dar.

Bemerkenswert ist, dass gerade das Zungenbein derjenigen eurystomen Ophidier, welche gewöhnlich, als besonders typisch ausgebildet, am meisten von den Lacertiliern entfernt werden, die relativ am wenigsten rückgebildeten Hyoide darbieten, dass ferner die peropode Familie der Boidae, die noch leidliche Reste von Gliedmassen bewahrt hat, einen weiteren Grad der Reduction des Zungenbeins aufweist und dass endlich, soweit bekannt, die angiostomen Familien das am meisten verkümmerte Hyoid besitzen. Somit eine erhebliche Discrepanz der verschiedenen morphologischen Merkmale dieser Abteilungen, die jedenfalls dazu mahnt, den Charakteren des Hyoides innerhalb der Ordnung der Schlangen keine massgebende taxonomische Bedeutung beizumessen. $\mathrm{Ob}$ es Ophidier gibt, bei denen: das Zungenbein völlig geschwunden ist, erscheint als möglich, ist aber bisher nicht nachgewiesen worden. Da, wo es Joh. Müller (31) vermisste (Tortrix, Typhlops, Rhinophis), wurde es später gefunden.

\section{Chelonia (Testudinata).}

Die Chelonier bilden eine meiner Ansicht nach von den Rhynchocephalia, Lacertilia und Ophidia ganz abseits stehende Urdnung der Reptilien, deren eigenartige Spezialisierung schon ihren frühesten Untersuchern aufgefallen und immer wieder von ihnen betont worden ist. Dabei haben zahlreishe hervorragende Zoologen und Anatomen der älteren Zeit, u. A. Meckel (23), Gegenbaur $(70,78,98)$ und Huxley (71), ihnen die primitivste Stellung unter den lebenden Reptilien zuerkannt und auf gewisse Ähnlichkeiten und Verwandtschaften mit den Amphibien, selbst mit den anuren Amphibien hingewiesen. Auch ich bin früher (74) diesen Anschauungen gefolgt, indem ich bei Bebandlung des Schultergürtels und der Schultermuskeln die Chelonier auf die Amphibien folgen liess, und erst nach ihnen die Lacertilier, habe mich aber weiterhin (00) überzeugt, dass die Lacertilier und der ihnen nahe verwandte Sphenodon auf diesem Organgebiete mehr primitive Züge darbieten als die. Chelonier, denen ich eine mittlere Entwickelungshöhe innerhalb der Reptilien zuschrieb. Und ich stehe mit dieser Auffassung nicht allein. Danach sind C. Rabl (03, 08, 10), Ribbing (07, 09), Sieglbauer (09) auf Grund der Charaktere des Schädels, sowie des Skeletes und der Muskulatur der Gliedmassen wieder für eine sehr primitive Stellung der Chelonier unter den lebenden Reptilien eingetreten. Von Seite der Paläontologen ist schon vor langer Zeit auf gewisse Ähnlichkeiten und Verwandtschaften mit den Rhynchocephaliern (Cope 70, 87, 89, Seeley 74, Boulenger 89 u. A.), namentlich aber mit den Sauropterygiern (Owen 39, Cope 71, 87, Baur 87, 88 u. v. A.) aufmerksam gemacht worden. Andere (z. B. Zittel 89 und Haeckel 95) fanden bei den Anomodontia nähere Anschlüsse, Osborn (03) vereinigte Cotylosauria, Anomodontia, Testudinata und Sauropterygia zu seiner Subclassis Synapsida und Jaekel (10) bildete aus den Cotylosauria, Theriodontia, Anomodontia, Testudinata und Monotremata die Klasse Paratheria, welche er zwischen Reptilia und Mammalia stellte. Weiter war wegen des gemeinsamen Merkmales der Stegocrotaphie des Schädels der Cotylosaurier und gewisser Chelonier eine Ableitung der Letzteren von den Ersteren, den primitivsten Formen unter den Reptilien, vertreten worden (Cope 92, 96, Baur 96, Woodward 98, Osborn 03, Broili 04, Williston 04, Case 05, Hay 08, Broom u. A.), und diese Phylogenese hatte durch die genauere Untersuchung neu aufgefundener gut erhaltener Reste des oberpermischen Cotylosauriers Eunotosaurus africanus (bei dem bereits Seeley 92 auf chelonierähnliche Züge hingewiesen) durch D. M. S. Watson (14) eine besondere Bestätigung erhalten. Wenn ich auch mit Siebenrock u. A. in der Stegokrotaphie gewisser Schildkröten (namentlich auch der fossilen Amphichelydia und der Cheloniidea) kein zweifelloses und durchgreifendes Kennzeichen einer primitiven, ursprünglichen Bildung erblicke, so erkenne ich doch die übrigens auch auf andere Merkmale gegründeten nahen Beziehungen der Chelonier zu Eunotosaurus an, erblicke aber darin noch keine beweisende Instanz für eine besonders tiefe Stellung der Schildkröten am Anfange der lebenden Reptilien.

Das Zungenbein der Chelonier haben zahlreiche Autoren untersucht, von denen hier Bojanus (1819-21), Cuvier (24, 36, 38), Meckel (29), E. Geoffroy St. Hilaire (32), Alessandrini (34), A. F. J. C. Mayer (35), Stannius $(46,56)$, Owen (66), Gegenbaur (70,98), Huxley (71), Brühl (80), Parker (80), Heude (80), Peters (82), Ludwig Ferdinand Prinz von Bayern (84), Gadow (88, 01), Boulenger (89), Hoffmann (90), Gaupp (99, 05), Oppel (00), Haller (04), Beddard (07), H. Fuchs (07, 12), Wiedersheim (09), Bütschli (10), Boas (11), Bender (11, 12), Kunkel (11, 12, 15), Ogushi (11, 13), Nick (12), Smith (14) und vor Allen Siebenrock $(98,00,06,13)$ hervorgehoben seien. Parker hat die Entwickelung älterer, Fuchs, Bender und Kunkel diejenige jüngerer Stadien bearbeitet; auch Siebenrock verdanken wir zahlreiche Angaben über die spätere (postembryonale) Entwickelung. Die Beziehungen zum columellaren Apparat wurden ausser von den eben genannten Untersuchern insbesonders von Peters (69), Gadow (88), Gaupp $(98,05)$ und Noack (07) behandelt, der Kehlkopf, zum Teil in seinem Verhalten zum Zungenbein, von zahlreichen Autoren, von denen ich hier nur Meckel (33), Mayer (35), Henle (39), Stannius (56), Owen (66), Huxley (71), Peters (82), E. Dubois (86), Gegenbaur (98), Göppert (99, 01), Siebenrock (99, 00, 06), Wiedershein (09), V. Schmidt (10,13), Bender (14) und Ogushi (14) nenne. Mayer (35) ist meines Wissens der Erste, der eine richtige Deutung des Kehlkopfes als Cricoid mit Arytaenoid und Nebenknorpeln gab, eine Deutung, die für fünfzig Jahre verschollen blieb und erst wieder mit Wiedersheim (83) und der ausgezeichneten Untersuchung von E. Dubois (86, der übrigens keine Kenntnis von Mayer's Arbeit hatte) wieder zu ihrer bleibenden Anerkennung kam. Der in der Nachbarschaft des Zungenbeins liegende, gemeinhin als "Entoglossum” bezeichnete Skeletteil fand gleichfalls manche Bearbeitungen und Besprechungen, von denen diejenigen von Cuvier (24, 36), Meckel (29), Alessandrini (34), 
posterior); die vorderen können entsprechend der Reduction der Cornua hyalia sehr zurückgebildet oder verschwunden sein. Von der Mitte des Vorderrandes erhebt sich der Processus lingualis, der gegenüber dem der Lacertilier in der Regel erheblich an Länge zurücktritt. Bei Testudo ist er relativ am längsten und besten, bei Chelone, Pelomedusa und einzelnen Vertretern der Trionychidae leidlich entwickelt; die Mehrzahl der Chelonier zeigt ihn nur schwach entfaltet und wenig vom Körper abgesetzt. Auch ist er derjenige Teil des Körpers, der meist am spätesten und bei vielen Arten nicht vollständig oder gar nicht verknöchert. Fuchs $(07)$ und Kunkel (12) beobachteten in frühen embryonalen Stadien wie bei dem Körper auch beim Proc. lingualis eine paarige Anlage.

Das Cornu hyale repräsentiert eine unbedeutende Bildung, die meist in Gestalt einer kleinen dreieckigen Knorpelplatte bindegewebig oder faserknorpelig mit dem Proc. lateralis anterior des Körpers verbunden ist; bei älteren Exemplaren von Emys orbicularis wird Verknöcherung beschrieben. Bei Platysternum, einigen Testudinidae (Nicoria, Testudo), den meisten Chelyidae (excl. Emydura) und den Trionychidae fehlt es oder wird es als fehlend angegeben; doch fanden es Ogushi (11) bei Trionyx sinensis und ich bei Tr. muticus als recht kleine Knorpelplatte. Bei jüngeren Embryonen von Emys orbicularis sahen Fuchs $(0 \%)$ und Kunkel $(11,12)$, dass es 'mit dem Körper in knorpeliger Continuität stand; Parker (80) beobachtete es bei einem älteren Embryo von Chelone mydas relativ etwas länger als bei einem nahezu reifen Jungen. Bender $(11,12)$ gelang bei Testudo graeca der wichtige Nachweis eines continuirlichen Verbandes des zu dieser frühen Zeit noch langen Hornes (Zungenbeinbogens) mit dem Interhyale und der Columella im Chondroblastem-Stadium; derselbe löste sich aber sehr bald, bereits vor der Jungknorpelbildung. Dieser Befund kennzeichnet genugsam, dass die Chelonier den ursprünglichen Zusammenhang des Hyoidbogens mit dem columellaren Apparat viel früher als die Lacertilier aufgegeben haben.

Das Cornu branchiale I. ist nach Umfang, Leistungsfähigkeit und früher Entwickelung das Haupthorn des Zungenbeins der Chelonier und zugleich der einzige Teil desselben, der bereits beim Embryo verknöchert. Es bildet im fertigen Zustande einen langen und kräftigen Knochenstab, meist von überwiegend rundlichem Querschnitt, bei nicht wenigen Vertretern aber im distalen Bereiche oder in seiner ganzen Länge (z. B. bei Chelys) auch abgeplattet. Sein Verlauf geschieht in caudal-lateraler Richtung, seltener geradlinig, meist im Bogen oder in winkeliger Knickung. Am Ende geht es in eine kurze knorpelige Epiphyse über. Hier ist es auch bei der Mehrzahl der Schildkröten mit einem besonderen, bereits Bojanus (1819-21) bekannten Knorpelstück, Epibranchiale I. (Siebenrock), verbunden, das nach Fuchs' und Kunkel's Untersuchungen an Emys orbicularis bereits im embryonalen Leben sich von dem Branchiale I. abgliedert, im Alter bei gewissen Chelyidae (Chelys, Hydraspis). auch verknöchern kann. Dieses Epibranchiale repräsentiert ein Gebilde, das auf primitive Verhältnisse der Chelonier hinweist.

Das Cornu branchiale II. fehlt keiner Schildkröte, steht aber hinsichtlich seiner erst postembryonal erfolgenden Verknöcherung hinter dem 1. Branchialhorn zurück. Auch ist es bei der Mehrzahl der Chelonier, namentlich bei den Abteilungen, die als die niedrigeren und ursprünglicheren anzusehen sind (Cryptodira), sowie bei den primitiveren Formen der Chelyidae (Chelodina, Emydura) kleiner oder wenigstens kürzer als das Cornu branchiale I. und zugleich einem grösseren Wechsel unterworfen als dieses. Gleich lang oder selbst grösser als das 1. Branchialhorn findet es sich dagegen bei der Mehrzahl der Pleurodira und Trionychidae, also bei denjenigen Schildkröten, welche als die höheren und einseitiger entwickelten angesprochen werden dürfen; hier zeigt es auch oft platte, selbst blattartige Configurationen. Seine Richtung und sein Verlauf zeigen eine gewisse Parallelität zu denen des Cornu branchiale I., von der aber sehr oft abgewichen wird. Bei gewissen Arten von Testudo, sowie bei Chelone und Dermochelys ist bisher noch keine. Verknöcherung gefunden worden; sie findet hier vielleicht im hohen, bisher noch nicht untersuchten Alter statt. Bei Emys orbicularis ossificiert das Horn früher als der Körper des Zungenbeins. Weitergehende und verhältnismässig früh einsetzende Verknöcherungen bieten die höheren Chelonier (Pleurodira und 
Trionychidae) dar, worüber, namentlich bei den Trionychidae, Siebenrock interessante Mitteilungen macht. Am Ende des. Hornes ist bei einigen höheren Sehildkröten (Chelodina, Emyda) gleichfalls ein separates kleines Skeletelement von Knorpel (Emyda) oder Knochen (Chelodina) von Siebenrock gefunden und als Epibranchiale II. bezeichnet worden. - Gegenüber den bei den Lacertiliern beobachteten: Vorkommnissen findet sich hier eine voluminösere, nicht zur secundären Sonderung in einen ventralen und dorsalen Teil und deren Entfernung von einander neigende und geringere Rückbildungen darbietende. Ausbildung des 2. Branchialhornes. Die primitiveren Chelonier zeigen dasselbe jedoch einfacher und in seiner Ausdehnung erheblich beschränkter als die primitiveren Formen unter den Lacertiliern; erst bei den höheren Schildkröten setzt die voluminösere und mit der Ossificierung geweblich mehr vorgeschrittene Gestaltung und Textur ein, so dass man diese - abgesehen von dem Epibranchiale II. - wohl richtiger als eine einseitige secundäre Weiterbildung, aber nicht als ein ursprüngliche Verhältnisse wahrendes Stehenbleiben aufzufassen hat.

Der ansehnliche, lange Zungenbeinkörper der Schildkröten bietet eine gute Stütze und einen bemerkenswerten Schutz für den. Anfang der Luftwege dar. Dieselben sind sicher und fest in seine concave dorsale Fläche eingebettet, derart, dass das. Cricoid nebst Aditus laryngis dem vorderen, der Anfang der Trachea dem mittleren und hinteren Abschnitt des Hyoides entspricht. Dieses Lageverhältnis ist zu dem der Lacertilier wegen der sehr verschiedenen Gestaltung beider Zungenbeinkörper nicht, leicht serial in Parallele zú bringen. Es entspricht aber einigermassen dem von Sphenodon, einigen Geckoniden und den Chamaeleonten, wo auch das Hyoid an der Deckung des Kehlkopfes und des Anfanges der Trachea Anteil hat. Dass man danach das Verhalten bei den Cheloniern nicht schlechtweg als ein rein primitives $\mathrm{zu}$ beurteilen habe, zeigen die Chamaeleontidae, welche eine recht primitiv scheinende Lagerung des Cricoides zum Zungenbein darbieten und dabei zugleich zu den höchsten. Lacertiliern gehören. Bender (14) hat aber auch hier bei Testudo graeca :Vorwanderungen des. Cricoides während der embryonalen Genese beobachtet. Dass es bei den Cheloniern nicht $\mathrm{zu}$ einer das Hyoid rostralwärts überschneidenden Vorwanderung der Luftwege kam, wird wohl durch den festen Verband des Anfanges derselben mit dem voluminösen Zungenbein bedingt; hierdurch wurden die bei gewissen Schildkröten recht bedeutend in ihrer Länge wachsende Trachea und ihre Äste gezwungen, sich in mannigfachen Windungen (über welche Siebenrock des Genaueren berichtet) zusammenzulegen, konnten aber nicht, wie bei den meisten Lacertiliern und Schlangen, in gerader Linie sich über das Zungenbein hinaus bis in den vorderen Bereich der Mundhöhle vorschieben.

Den Cheloniern kommt in der vorderen und ventralen Nachbarschaft des Zungenbeins noch ein eigenartiger, viel untersuchter und viel besprochener Skeletteil zu, das sogenannte Entoglossum, welches von Bojanus (1819-21) bei Emys orbicularis ursprünglich als Glandula salivalis sublingualis bezeichnet, dann aber von Cuvier (24), Meckel (29) und weiteren Untersuchern je nach der untersuchten Art als faseriges, faserknorpeliges, hyalinknorpeliges und zum Teil verknöchertes Skeletelement erkannt wurde. Cuvier nannte es "Os de la langue", Meckel "Kleiner mittlerer Unterzungenknorpel", die folgenden Untersucher, an Cuvier anknüpfend, "Pars lingualis, Cartilago lingualis, Os linguale”. Stannius (1846) führte die verhängnisvolle Bezeichnung "Pars lingualis s. Cartilago entoglossa” ein, obwohl er den Skeletteil richtig als eine unter und zum. Teil vor dem Zungenbeinkörper gelagerte und ihm nur lose angeheftete Platte charakterisierte, und die nach ihm darüber handelnden Autoren übernahmen grösstenteils die unpassende, weil eine Lage innerhalb der. Zunge ausdrückende Bezeichnung als „Pars entoglossa, Os entoglossum oder Entoglossum (Entoglosson)".

Die Platte liegt in Wirklichkeit nicht innerhalb der, Zunge, hat auch nichts mit dem Zungenbein zu tun, sondern liegt ihm nur ventral auf, durch lockeres Bindegewebe und einige Muskulatur mit ihm verbunden. Siebenrock (98) bezeichnete sie als eine Eigentümlichkeit der Schildkröten, die bei keiner Gattung derselben fehlen dürfte. Verschiedene neuere Autoren (Gegenbaur 98, Gaupp 05, Bütschli 10, Schimkewitsch 10 u. A.) gaben ihren Zweifeln an der Zugehörigkeit zur Zunge oder zum Zungenbein Ausdruck (,sogenanntes Entoglosşum") und Andere (Fuchs 07, Bender 11, 12, Nick 12) leugneten namentlich auch auf Grund ihrer ontogenetischen Untersuchungen jede direkte Beziehung zum Hyoid. Die Bezeichnung „Entoglossum” blieb aber bis auf den heutigen Tag bestehen.

Mir erscheint es nur korrekt, diesen irrigen Terminus, der ausserdem zu Verwechselungen mit dem wirklich zum Zungenbein gehörigen Processus lingualis s. entoglossus (Entoglossum) der Lacertilier und anderer Reptilien, sowie der Vögel u.s. w. führt, zu streichen und zugleich unter Wahrung der Priorität zu der von Meckel 17 Jahre vor Stannius eingeführten Bezeichnung "Unterzungenknorpel” zurückzukehren, den Skeletteil also Hypoglossum zu benennen. 
Das Hypoglossum ist ein bisher nur bei den Schildkröten und hier noch nicht bei allen Vertretern derselben gefundener Skeletteil, der ganz unabhängig vom Hyoid und erheblich später als dasselbe auf bindegewebiger Grundlage entsteht (Fuchs $0 \%$ vermisste es noch bei älteren Embryonen und jüngeren Tieren von Emys orbicularis, Bender 11, 12 fand es bei Testudo graèca sehr spät auftretend, und Nick 12 verfolgte seine weitere Entwickelung bei verschiedenen Cheloniern), danach in sich einzelne Knorpelinseln ausbildet, die weiterhin zusammenfliessen und schliesslich bei einzelnen Arten partiell verknöchern können. Siebenrock verdanken wir die ausführlichsten Untersuchungen über das ausgebildete Hypoglossum, und gern stimme ich seiner Behauptung bei, dass es ein den Schildkröten eigentümliches Gebilde sei, das wahrscheinlich bei keinem Chelonier fehle, aber bei einigen Arten erst so spät zur Ausbildung komme, dass es bisher bei nicht ganz ausgewachsenen Individuen derselben vermisst worden sei. Brühl (80) versuchte eine Vergleichung mit dem Glosso-hyale und Urohyale der Fische, die als verunglückt zu bezeichnen ist, denn diese Skeletelemente gehörén $\mathrm{zu}$ den wirklichen und tiefer gelegenen Teilen des hyobranchialen Apparates.

Bei den Cryptodira bildet das Hypoglossum eine mässig grosse ovale Platte von festem Bindegewebe oder Faserknorpel oder Hyalinknorpel, welche Gewebe auch gemischt vorkommen können. Unter den Cheloniidea wurde bei Dermochelys bei einem bereits recht alten (1 $1 \frac{1}{2}$ Meter langen) Exemplar von Nick (12) noch kein Hypoglossum gefunden, und auch bei den Cheloniidae scheint es recht spät zur Ausbildung zu kommen. Die höher stehenden Pleurodira und Trionychidae dagegen zeigen es in grösserem Volumen bei überwiegender Ausbildung von Hyalinknorpel, und bei gewissen Chelyidae (Chelys, Hydromedusa und Hydraspis) kommt es in der Knorpelplatte zu einem Paar mehr oder minder ausgedehnter Verknöcherungen. Die Gestalt des Hypoglossum wechselt hierbei bei den verschiedenen Cheloniern mannigfaltig. Bei im Ganzen ovalem Umriss kann es mancherlei Fortsatzbildungen, Verschiedenheiten des caudalen und rostralen Endes und unregelmässige bezw. nicht scharf umrissene Konturen zeigen, auch von mehr oder mindex zahlreichen feinen Löchern durchbohrt und in durch Bindegewebe verbundene getrennte Knorpelstücke gesondert sein, was alles für die Entstehung aus ursprünglich isoliert auftretenden Knorpelkernen spricht. Die paarigen verknöcherten Stellen fand ich bei Hydromedusa tectifera annähernd kreisförmig; bei Chelys fimbriata sind sie seit Cuvier als ausgedehnt halbmondförmig bekannt.

Zusammenfassend kann vom Hyoid der Chelonier gesagt werden, dass es nicht tiefer steht als das Zungenbein der Lacertilier. Die sehr frühe und weit fortgèschrittene Rückbildung des hyalen Hornes entfernt es erheblich von Sphenodon und den meisten primitiven Abteilungen der Lacertilier und lässt graduell nur an diejenigen Saurier denken, die wie gewisse Amphisbaenidae, Acontias und Typhlosaurus auch einen erheblichen Teil ihres Cornu hyale eingebüsst haben. Dies sind aber unter gleichzeitiger Verkümmerung der Gliedmassen und unter Verlängerung des Gesamtkörpers zu schlangenähnlichen Sạuriern umgebildete Formen, die also für eine Vergleichung mit den Schildkröten nicht in Frage kommen. Die einigermassen gleichartige Gestaltung der vorderen und hinteren Branchialhörner die auf den ersten Anblick dem Zungenbein der Chelonier ein primitives Gepräge zu verleihen scheint, hält bei genauerer Betrachtung nicht Stand, da bei den niedrigeren Cheloniern das zweite Branchialhorn hinter dem ersten zurücksteht und erst bei den höheren Abteilungen eine voluminösere Ausbildung erlangt. Auch bietet das Cornu branchiale II. der in dieser Hinsicht primitivsten Lacertilier eine, entsprechend dem leichteren Körper derselben zwar schlankere, aber doch ausgedehntere Entfaltung dar. Dass es bei dem Hyoid der Schildkröten auch zu einer vorgeschrittenen Verknöcherung des Körpers und des 2. Branchialhornes kommen kann, die Sphenodon und den Lacertiliern noch durchaus abgeht, ist gleichfalls Kennzeichen einer höheren Differenzierung. Die beiden von den Branchialia abgegliederten Epibranchialia I. und II. lassen sich vielleicht als primitivere Gebilde auffassen; doch erzählt ihre Kürze und Kleinheit von bereits vorgeschrittenen Verkümmerungen, während die dorsalen Teile des 
Cornu branchiale II. vieler Lacertilier, die auch ein solches, hier unselbständiges Epibranchiale II. in sich enthalten mögen, eine weit grössere dorsale Entfaltung darbieten. Jedenfalls muss hier noch weiter untersucht werden. Die ursprüngliche Anlage des Zungenbeinkörpers der Chelonier ist als eine relativ tiefstehende zu beurteilen. Das Hypoglossum ist eine specifische Bildung, die erst innerhalb des Ordo Chelonia eine späte Entwickelung genommen hat, dem Hyoid fern steht und snnach mit den Zungenbeinen anderer Reptilien nicht zu vergleichen ist.

\section{Crocodilia (Loricata, Eusuchia).}

Über die Stellung des Ordo Crocodilia innerhalb der lébenden Reptilien besteht'seit geraumer Zeit kein Zweifel mehr, von den Lacertilia (Sauria squamata), mit denen sie früher als Sauria loricata vereinigt wurden, sind sie auf Grund der monimostylen Beschaffenheit ihres Kieferapparates (Stannius) und vieler anderer Differenzen in ihrem sonstigen Bau längst abgetrennt. Sie gelten als höchste Abteilung der lebenden Reptilien und zeigen gegenüber allen anderen Ordnungen derselben eine ausgesprochene Isolierung:

Entsprechend der geringen Anzahl in der Jetztzeit noch zurückgebliebener Arten der aus nur 1 Familie (Crocodilidae) bestehenden Ordnung ist die Zahl der Untersuchungen über das Hyoid derselben eine beschränkte; auch hat dessen einfacher Bau den Autoren keine ausgedehnte Gelegenheit zu Fragstellungen gegeben.

Von den Untersuchern des Zungenbeines hebe ich Cuvier (24,36,38), Meckel (29), Mayer (35), Duvernoy (35), Stannius (56), Brühl (62), Salverda (63), Owen (66), Huxley (71), Parker (83), Ludwig Ferdinand Prinz von Bayern (84), Hoffmann (90), Gegenbaur (98), Göppert (99, 03), Gaupp (05), Wiedersheim (09), Bütschli (10) und Shiino (14) hervor, von denen Parker ältere und Shiino jüngere embryologische Stadien auf die Ontogenese des Zungenbeins bearbeiteten (in Rathke's nachgelassenem Werke 66 findet sich nur eine wenig brauchbare Abbildung des embryonalen Hyoides). Die Beziehungen zu dem columellaren Apparat wurden namentlich von Peters (68, 70), Huxley (69), Gadow (88), Killian (90), Versluys $(98,03)$, Gaupp $(99,05)$ und Shiino (14) erörtert; über den Kehlkopf und sein Verhältnis zum Zungenbein haben namentlich Cuvier (24), Meckel (33), Mayer (35, auch hier mit erster richtiger Deutung des Kehlkopfes), Henle (39), Rathke (66) und Göppert (99, 03) gehandelt.

Meine eigene Untersuchungen beschränken sich auf wenige Species. Zusammen mit denen der früheren Bearbeiter verfüge ich nur über die Kenntnis von 9 Arten, von denen einige in mehreeren Exemplaren beobachtet wurden.

Das Zungenbein der Crocodilier ist von sehr einfacher Art und weicht von den Hyoiden der zuvor behandelten Reptilien durchaus ab. Es besteht aus einer recht ausgedehnten ventralwärts convexen Knorpelplatte, welche im Wesentlichen den Körper darstellt und vorn, an der Seite und hinten einige verschieden ausgeprägte Vorragungen zeigt. Seitlich geht vom Körper das grösstenteils verknöcherte Cornu bianchiale I. ab.

Der Körper bildet eine in der Länge und Breite recht ansehnliche, aber nicht sehr starke knorpelige Platte, in deren dorsaler Concavität der Kehlkopf (Cricoid und Arytaenoid) und der Anfang der Trachea eingelagert ist. Bei alten Tieren soll die Platte nach Cuvier in ihrem hinteren Bereiche verknöchern. Der Körper ist in seinem vorderen, vor der Einlenkung der Hörner gelegenen Bereiche breiter als im hinteren. Sein vorderer Umriss bildet bei Crocodilus eine einfache nach vorn convexe bogenförmige Linie; bei Alligator ist dieselbe von einem (oder zwei) Paar rundlicher Incisuren unterbrochen. Beim Embryo von Crocodilus porosus fand Shiino in der Mitte des vorderen Randes noch einen unbedeutenden Fortsatz, den er mit Recht als Processus lingualis anspricht; später wird derselbe ganz zurückgebildet. An den vorderen Rand schliesst sich unmittelbar im Bogen der Anfang des lateralen Randes an, der vor der Einlenkung des Cornu branchiale I. eine breite Vorragung zeigt, die Shiino (14) als Rest eines mit dem Körper verschmolzenen Cornu hyale deutet; ob mit Recht, kann ich mit den gegebenen Materialien nicht entscheiden. Zurückzuweisen ist aber die Behauptung von Brühl (62), der in den schmalen Knorpelzungen zwischen den beiden paarigen Einschnitten des Vorderrandes die Cornua hyalia erblickt. Der hintere Bereich des Körpers (hinter der Einlenkung der I. Branchialhörner) ist schmäler als der vordere und läuft caudalwärts in einen rechten und linken Zipfel aus, die mit Wahrscheinlichkeit als Reste der Cornua branchialia II. aufzufassen sind.

Die Existenz eines Cornu hyale bei ausgebildeten Tieren erscheint mir, wie angegeben, noch nicht gesichert. Die Möglichkeit, dass ein solches in früher Vorzeit existierte und sich als Hyoidbogen bis zum columellaren Apparat erstreckte, ist natürlich anzunehmen; allein dieser Hyoidbogen hat, wie schon Parker (83) mit Wahrscheinlichkeit dargetan, namentlich durch die Ausbildung des ansehnlichen Processus retroarticularis des Unterkiefers sehr früh eine völlige Unterbrechung erlitten und ist mit dem unteren Ende seiner dorsalen Strecke 
mit diesem Fortsatz verschmolzen, während die von da bis zum Hyoidkörper reichende ventrale Strecke - möglicherweise mit Ausnahme des letzten in die Seitenwand des Körpers aufgenommenen ventralen Endes des Bogens - in Rückbildung trat. Shiino scheint diesen Entwicklungsgang bei seinen Embryonen verfolgt zu haben; es ist mir aber nicht ganz klar geworden, wie viel von seiner Darstellung wirklich beobachtet, wie viel von ihm erschlossen worden ist.

Das knöcherne Cornu branchiale I. ist mit dem lateralen Rande des Körpers, mehr vorn bei Crocodilus, mehr hinten bei Alligator, gelenkig. verbunden und geht in überwiegend caudaler Richtung nach hinten, wobei es zugleich in seinem weiteren Verlaufe infolge von winkeliger Knickung lateral- und dorsalwärts sich wenden kann. An seinem freien Ende besitzt es eine knorpelige Epiphyse. Beim jungen Eimbryo besteht es durchweg aus Knorpel und hängt continuirlich mit dem Seitenrande des Körpers zusammen (Shiino).

Das knorpelige Cornu branchiale II. ist, wie schon angegeben, wohl in den beiden kurzen Hervorragungen des hinteren Randes des Körpers zu finden.

Das Verhalten des Hyoideș zu den Luftwegen nähert sich den Beziehungen bei den Cheloniern, indem der ausgedehnte Hyoidkörper dem Kehlkopf (Cricoid) ausreichenden Schutz gewährt; das Zungenbein ragt sogar vorn beträchtlich über das Cricoid vor, indem die Lage der Stimmritze ungefähr der Abgangsstelle des 1. Branchialhorns entspricht. Schon Cuvier (24) hat angeführt, dass der Zungenbeinkörper der Crocodile die Functionen des Thyreoides und der Epiglottis der Säugetiere erfülle, und Göppert (03) hat den damit zum Teil gegebenen Schutz gegen Eindringen von Wasser in die Luftwege genauer erläutert. Natürlich ist hierbei an keine morphologische Vergleichung mit Thyreoid und Epiglottis der Mammalia zu denken. Ob die erhebliche rostrale Vorragung des Hyoidkörpers über den Kehlkopf einer secundären. Vergrösserung des ersteren oder einem Zurückbleiben bezw. Zurückweichen der Luftwege seine Ausbildung verdankt, dürfte vielleicht durch die genauere ontogenetische Untersuchung dieses Verhältnisses zu entscheiden sein.

Im Ganzen kennzeichnet sich das Hyoid der Crocodilier als ein Gebilde, welches sich durch frühe Trennung des Hyoidbogens in seinem ventralen -Gebiete, secundäre Verbindung des oberen Teiles mit der Mandibula und durch völliges Aufgehen des ventralen Endes in den Körper bezw. durch gänzlichen Schwund dieses Endes von dem Ausgangspunkte seiner Entwickelung weit entfernt hat. In seiner geweblichen Textur hat es die ursprünglichen $\mathrm{Zu}-$ stände mehr gewahrt. Der Körper des Hyoides hat sich vergrössert, alle anderen Teile haben sich vermindert und, abgesehen vom 1. Branchialhorn, ihre Selbständigkeit dem Körper gegenüber eingebüsst. Hierdurch steht das Zungenbein der Crocodile von denen der anderen lebenden Reptilien entfernt und auf einsamer Höhe. Mit dèm Hyoid der Chelonier hat es sehr wenig gemein, mit dem von Sphenodon und den Lacertiliern nicht viel mehr; doch deuten einzelne Züge, z. B. auch das Verhalten des Cornu branchiale II., darauf hin, dass seine Wurzel der Wurzel der beiden zuletzt erwähnten Reptilienordnungen etwas näher stand als derjenigen der Schildkröten.

\section{Zusammenfassung}

Unter den lebenden Reptilien nimmt das Zungenbein von Sphenodon auf Grund der allerdings nicht immer vorhandenen ursprünglichen Erhaltung des Hyoidbogens die primitivste Stelle ein, steht aber in der ziemlich weit vorgeschrittenen Rückbildung des dorsalen $A b$ schnittes des Cornu branchiale II weiter von seinem Ausgangspunkte ab als die primitiveren Lacertilier, von denen die Eublepharidae und Xantusiidae dieses Horn entweder in seiner Vollständigkeit oder nach der Trennung in den ventralen und dorsalen Teil in diesen beiden Abschnitten in einer Vollkommenheit gewabrt haben, welche allen anderen lebenden Reptilien abgeht und selbst Anklänge an die Verhältnisse bei den Amphibien erkennen lässt. Unter den Lacertiliern bieten die primitiveren Geckonidà, Eublepharidae und Uroplatidae, sowie die Amphisbaenidae auch noch eine Verbindung mit Teilen des columellaren Appa- 
rates dar, jedoch nicht mehr in dem ursprünglichen Verhalten wie bei Sphenodon. Die übèrwiegende Mehrzahl der Lacertilier hat diese Verbindung ganz aufgegeben, zeigt auch am hyalen und am 2. branchialen Horn Rückbildungen sowie einseitige Umbildungen verschiedener Grade, welche diese Vertreter der Ordnung noch weiter vom ursprünglichen Verhalten entfernen, jedoch nur zum kleinsten Teil als Kennzeichen einer höheren Entwickelungsstufe zu bewerten sind. Die Ophidier zeigen in ihrem Zungenbein einen ganz einseitigen Reductionsprocess vom- Lacertiliertypus, welcher das Hyoid derselben auf die tiefste Stufe seiner Leistungsfähigkeit bringt. Weit voluminöser und vollkommener ist das Zungenbein der Chelonier gestaltet und offenbart in der Ausbildung seines Körpers und der Existenz der Epibranchialia I. und II. primitive Züge, während die Reduction seines Cornu hyale, seine ganze gewebliche, zu ausgedehnten Ossificationen neigende Beschaffenheit und auch das Verhalten des Cornu branchiale II. (welches den Anschein eines primitiven Verhaltens gewährt, in Wirklichkeit aber auf einseitiger Weiterentwickelung beruht) im Vergleich mit dem Hyoid der Lacertilier ein Plus von höheren Merkmalen zu erkennen gibt. Zudem steht das Hyoid der Chelonier in der Reihe der lebenden Reptilien ganz isoliert. Bei den Crocodiliern kennzeichnet die weitgehende (vielleicht vollständige) Rückbildung des hyalen Hornes und die Aufnahme der reducierten Elemente des Cornu hyale und Cornu branchiale II. in den Hyoidkörper, insbesondere auch die bedeutende Vergrösserung des Körpers einen besonderen Entwickelungsgang, der das Crocodilierhyoid isoliert und ziemlich hoch stellt, jedoch auch unter Berücksichtigung seiner weniger hochstehenden geweblichen Beschaffenheit an entfernte Ähnlichkeiten mit den Lacertiliern denken lässt.

Für die tiefere und höhere Stellung der verschiedenen Vertreter der lebenden Reptilien haben wir namentlich im Skeletsystem des Kopfes, der Wirbelsäule, der Extremitätengürtel und freien Gliedmassen, im Muskel- und Nervensystem und in anderen Organen Kriterien, welche höher zu bewerten sind als das Zungenbein. Dieses darf aber nicht gering geachtet werden. Auch soll man immer eingedenk sein, dass nur in den wenigsten Tierkörpern die verschiedenen Organsysteme eine gleiche Höhe der Entwickelung darbieten. Fast immer tritt im Organismus je nach seiner funktionellen Beanspruchung bald dieses, bald jenes Organ in den Vordergrund, und dementsprechend kommen andere nicht zur gleichen Geltung, bleiben stehen oder gehen auch in Rückbildung. Gewöhnlich sind selbst in demselben Organ Züge der höheren und der minderen Ausbildung neben einander vertreten. Da bedarf es einer sehr vorsichtigen und umsichtigen Abwägung der morphologischen Bedeutung der höheren und tieferen Merkmale, um zu entscheiden, ob. diese oder jene den Ausschlag für die tiefere oder höhere Stellung des einen oder des anderen Tieres geben. Das Gleiche gilt für die Bemessung der näheren oder ferneren gegènseitigen Verwandtschaften. Dies sind alles selbstverständliche und zu ungezählten Malen exöṛterte Wahrheiten, jedoch in ihren speziellen Anwendungen nicht immer leicht zur Geltung zu bringen.

Soweit es sich zur Zeit übersehen lässt, bildet die Configuration des Zungenbeins keinen Factor, welcher sich den auf anderen Wegen gewonnenen Anschauungen über die gegenseitigen Verwandtschaften und tieferen und höheren Stellungen der verschiedenen Reptilienordnungen und ihrer Unterabteilungen feindlich entgegenstellt. Man darf mit ihnen rechnen als mit einer diese Anschauungen im Grossen und Ganzen ergänzenden und zum Teil sie selbst corrigierenden Instanz. 